\title{
Entanglement relaxation of poly(1-butene) and its copolymer with ethylene detected in conventional shear rheometer and quartz resonator
}

Article

Accepted Version

Liu, C., Liu, P., Chen, Q., Du, B. and Wang, Z. (2019)

Entanglement relaxation of poly (1-butene) and its copolymer with ethylene detected in conventional shear rheometer and quartz resonator. Journal of Rheology, 63 (1). pp. 167-177. ISSN 0148-6055 doi: https://doi.org/10.1122/1.5051041 Available at https://centaur.reading.ac.uk/81512/

It is advisable to refer to the publisher's version if you intend to cite from the work. See Guidance on citing.

To link to this article DOI: http://dx.doi.org/10.1122/1.5051041

Publisher: Society of Rheology

All outputs in CentAUR are protected by Intellectual Property Rights law, including copyright law. Copyright and IPR is retained by the creators or other copyright holders. Terms and conditions for use of this material are defined in the End User Agreement. 


\section{CentAUR}

Central Archive at the University of Reading

Reading's research outputs online 


\section{Entanglement Relaxation of Poly(1-butene) and its Copolymer with}

\section{Ethylene Detected in Conventional Shear Rheometer and Quartz \\ Resonator}

Chang Liu, Peiru Liu, and Quan Chen*

State Key Laboratory of Polymer Physics and Chemistry, Changchun Institute of Applied Chemistry, Chinese Academy of Sciences; University of Chinese Academy of Sciences, Changchun 130022, China and University of Chinese Academy of Sciences, Beijing 100049, China

\section{Binyang $\mathrm{Du}^{*}$}

MOE Key Laboratory of Macromolecular Synthesis and Functionalization, Department of Polymer Science \& Engineering, Zhejiang University, Hangzhou 310027, China

Zuowei Wang

School of Mathematical, Physical and Computational Sciences, University of Reading, Whiteknights, Reading RG6 6AX, U.K.

*QC: qchen@ciac.ac.cn; BD: duby@zju.edu.cn 


\section{Abstract:}

This study examined linear viscoelasticity (LVE) of stereo-regular poly(1-butene) (PB) and its random copolymer with polyethylene (PBE), with number fraction of ethylene comonomer $\leq 10 \%$, using a conventional shear rheometer combined with a quartz resonator. This combination resulted in the detection of LVE in a broad frequency range from Rouse region to terminal relaxation. LVE thus determined was fit to the double reptation model through utilizing inputting molecular weights determined by gel permeation chromatography equipped with refractive index and two-angle laser light scattering monitors, and the segmental time and entanglement molecular weight as the two fitting parameters. Quantitative prediction was finally achieved when the two fitting parameters changed systematically by the increase of ethylene content; a decrease of segmental time quantified a plasticizing effect and a decrease of entanglement molecular weight reflected enhanced chain flexibility. The entanglement molecular weight of the PB samples was found to be lower than those reported for stereo-irregular PB, suggesting the important effect of stereo-regularity on chain flexibility. 


\section{Introduction}

Stereo-regular isotactic poly(1-butene) (PB) and its copolymers with ethylene (PBE) are widely used as feed pipe, owing to tolerance against wide temperature shifts, high yield stress, and strong chemical resistance. Commercially available PB and PBE are usually synthesized with the Ziegler-Natta catalysts. The crystallization temperature is usually much higher than that of glass transition when the ethylene content is less than $10 \%$, which results in a narrow temperature window for measuring melt rheology. [1-3]

To address this problem, one pathway would reduce the stereo-regularity in order to suppress crystallization. For example, Graessley and coworkers synthesized atactic PB and PE copolymers by hydrogenating the monodispersed polybutadiene samples synthesized through anionic polymerization, thereby varying PB content from $0 \%$ to $100 \%$ by tuning a ratio between the 1,4 and 3,4 additions. They then examined the linear viscoelasticity (LVE) of these atactic samples with varied PE content from $1 \%$ to $85 \%$ [4-6] to determine the plateau moduli $G_{\mathrm{e}}$ and, accordingly, the entanglement molecular weight $\left(M_{\mathrm{e}}\right)$ of these samples from

$$
M_{\mathrm{e}}=\rho R T / G_{\mathrm{e}}
$$

where $M_{\mathrm{e}}$ was evaluated to be $13,500 \mathrm{~g} / \mathrm{mol}$ for pure PB and decreased systematically with the increase of PE fraction, owing to the enhancement of chain flexibility. In addition, Graessley and coworkers noted an important plasticizing effect of the ethylene comonomers. [4-6] Namely, $T_{\mathrm{g}}$ reduced with increased ethylene content. Nevertheless, it remains unclear how $M_{\mathrm{e}}$ and $T_{\mathrm{g}}$ change with stereo-regularity.

To gain more insight into this question, an alternative pathway involves extending the window of time scales of the LVE measurements. Toward this end, Friedrich and coworkers 
recently demonstrated that the use of a conventional shear rheometer (detecting a frequency range of $\sim 10^{-2}-10^{2} \mathrm{rad} / \mathrm{s}$ ), a piezoelectric rotary vibrator (PRV, detecting a frequency range of $\left.\sim 1-10^{4} \mathrm{rad} / \mathrm{s}\right)$, and a quartz resonator $\left(\mathrm{QR}\right.$, detecting a frequency range of $\left.\sim 10^{8} \mathrm{rad} / \mathrm{s}\right)$ enables the detection of LVE from the Rouse regime to terminal relaxation for semi-crystalline PE and poly (vinyl acetate) (PVAc) copolymers. [7] This protocol is particularly useful when the ethylene content is above $60 \%$, the point at which crystallization of PE disturbs conventional LVE measurements at low $T$, in turn, enabling QR to effectively extend LVE measurements to the glassy-to-rubbery transition region.

The present study will examine linear viscoelasticity for stereo-regular PB and PBE samples by combining conventional rheology and QR. [4-6; 8] Although this experimental approach is arguably very similar to that of Friedrich and coworkers, their work is extended herein by the addition of a new data analysis protocol. We utilized molecular weight (MW) and its distribution directly obtained from gel permeation chromatography equipped with refractive index and light scattering monitors as inputting parameters, leaving only entanglement molecular weight $\left(M_{\mathrm{e}}\right)$ and segmental time $\left(\tau_{0}\right)$ as the fitting parameters. This approach enables quantitative prediction of LVE. Comparison of the entanglement molecular weight $M_{\mathrm{e}}$ of stereo-regular $\mathrm{PB}$ in this study and that of stereo-irregular PB studied by Graessley and coworkers suggests the important role of stereo-regularity in determining the chain flexibility. [9]

\section{Experimental}

\subsection{Samples}


All PB and PBE samples were produced by LyondellBasell Industries with the brand name listed in Table 1. For simplicity, we renamed the samples based on the molecular weights and fraction of ethylene monomers, as also shown in Table 1; for example, PB0800 is renamed as PB121, meaning that this PB sample has $M_{\mathrm{w}}=121 \mathrm{~kg} / \mathrm{mol}$, and PB8510 is renamed as PBE203-9.9\%, meaning that this PBE copolymer sample has $M_{\mathrm{w}}=203 \mathrm{~kg} / \mathrm{mol}$ and a molar fraction of ethylene monomers $f_{\mathrm{PE}}=9.9 \%$. Most commercially available PBE samples contain high content of stereo-regular PB owing to their application values.

Tacticity of the three PB samples was characterized via ${ }^{13} \mathrm{C}$ nuclear magnetic resonance. Meso and racemic placements consisted of two adjacent monomers oriented in the same and opposite directions, respectively. ${ }^{13} \mathrm{C}$ enables characterization of fractions of isotactic $(\mathrm{mm})$, heterotactic $(\mathrm{mr}$ or $\mathrm{rm})$, and syndiotactic (rr) triads, with $[\mathrm{mm}]+[\mathrm{mr}]+[\mathrm{rm}]+[\mathrm{rr}]=1$. Tacticity is defined as $[\mathrm{m}]=[\mathrm{mm}]+0.5([\mathrm{mr}]+[\mathrm{rm}])$. Details of these parameters are summarized in Table 2.

Table 1. Important parameters of PB and PBE samples

\begin{tabular}{cccccccc}
\hline Name & Code & $f_{\mathrm{PE}}$ & $M_{\mathrm{n}}(\mathrm{g} / \mathrm{mol})$ & $M_{\mathrm{w}}(\mathrm{g} / \mathrm{mol})$ & $M_{\mathrm{w}} / M_{\mathrm{n}}$ & $T_{\mathrm{g}}\left({ }^{\mathrm{b}} \mathrm{C}\right)$ & $T_{\mathrm{g}}{ }^{\mathrm{c}}\left({ }^{\circ} \mathrm{C}\right)$ \\
\hline PB0800 & PB121 & $0 \%$ & 47,000 & 121,000 & 2.6 & -26 & -29 \\
PB0400 & PB238 & $0 \%$ & 96,000 & 238,000 & 2.5 & -25 & -28 \\
PB0110 & PB742 & $0 \%$ & 227,000 & 742,000 & 3.3 & -22 & -27 \\
PB8640 & PBE552-1.5\% & $1.5 \%^{\mathrm{a}}$ & 160,000 & 552,000 & 3.5 & -27 & -31 \\
PB8220 & PBE414-4.3\% & $4.3 \%^{\mathrm{a}}$ & 151,000 & 414,000 & 2.7 & -32 & -34.5 \\
PB8510 & PBE203-9.9\% & $9.9 \%^{\mathrm{a}}$ & 77,000 & 203,000 & 2.6 & -35 & -35.5 \\
\hline
\end{tabular}

a: Reported in Ref $[10 ; 11]$

b: Obtained from the WLF analysis 
c: Obtained from the DSC measurements of crystallized samples

Table 2: Tacticity of the PB samples

\begin{tabular}{cccccc}
\hline Name & Code & {$[\mathrm{mm}]$} & {$[\mathrm{mr}]+[\mathrm{rm}]$} & {$[\mathrm{rr}]$} & $\begin{array}{c}{[\mathrm{m}]} \\
\text { (tacticity) }\end{array}$ \\
\hline PB0800 & PB121 & 0.960 & 0.020 & 0.020 & 0.970 \\
PB0400 & PB238 & 0.915 & 0.054 & 0.0311 & 0.942 \\
PB0110 & PB742 & 0.954 & 0.032 & 0.014 & 0.970 \\
\hline
\end{tabular}

2.2 Gel Permeation chromatography (GPC)

Molecular weight distribution of PB and PBE samples was characterized by high-temperature gel permeation chromatography (HT-GPC) analysis with PL-GPC 220 (Agilent), and the instrument was equipped with a two-angle $\left(15^{\circ}\right.$ and $\left.90^{\circ}\right)$ laser light scattering (TALLS) detector, a viscometer, and a differential refractive index (DRI) detector. Three PLgel $10 \mu \mathrm{m}$ MIXED-B LS columns (300x7.5mm) were used for the separation.

The TALLS and the DRI detectors were calibrated with standard polystyrene (PS) samples. 1,2,4-trichlorobenzene stabilized with $5 \times 10^{-4} \mathrm{~g} / \mathrm{mL}$ 2,6-di-tert-butyl-4-methylphenol (BHT) was used as the eluent. All measurements were performed at $150^{\circ} \mathrm{C}$ with an eluent flow rate of $1.0 \mathrm{~mL} / \mathrm{min}$. Polymer solution at a concentration of about $1.0 \mathrm{mg} / \mathrm{mL}$ was injected into the multi-detector GPC system via an autosampler with an injection volume of $200 \mu \mathrm{L}$. The refractive index increment $d n / d c$ of all studied PB and PBE samples was determined to be $-(0.097 \pm 0.001) \mathrm{mL} / \mathrm{g}$.

Cirrus software was used for data acquisition and analysis. For each elution fraction $i$, the molecular weight $M_{\mathrm{i}}$, radius of gyration $R_{\mathrm{g}, \mathrm{i}}$, intrinsic viscosity $[\eta] \mathrm{i}$, and concentration $C_{\mathrm{i}}$ 
were obtained. Then the weight average and number average molecular weights, $M_{\mathrm{w}}$ and $M_{\mathrm{n}}$, of the samples could be calculated with established absolute calibration curves, and results are summarized in Table 1.

\subsection{DSC measurement}

Thermal properties of the samples were measured with both conventional differential scanning (DSC) calorimeter (TA Instruments Q20) and Flash DSC. For conventional DSC measurements, specimens weighing 5-8mg, as cut from granules, were sealed in aluminum pans. The DSC equipment was calibrated using indium and tin. All experiments were performed under the protection of nitrogen atmosphere flowing at a rate of $50 \mathrm{~mL} / \mathrm{min}$.

To determine the glass transition of the crystallized sample, the samples were first annealed in the DSC instrument at $180^{\circ} \mathrm{C}$ for $10 \mathrm{~min}\left(>>\right.$ equilibrium crystallization point of $146^{\circ} \mathrm{C}$ of $\mathrm{PB}$ [29]) to remove thermal and flow histories, followed by cooling down to different temperature $T_{\mathrm{c}}$ and annealing there for $25 \mathrm{~min}$ for isothermal crystallization $\left(T_{\mathrm{c}}=90^{\circ} \mathrm{C}\right.$ for all PB samples, $70^{\circ} \mathrm{C}$ for PBE414-4.3\% and PBE203-9.9\%, and $40^{\circ} \mathrm{C}$ for PBE203-9.9\%, at which the isothermal crystallization was finished within 25min). After isothermal crystallization, the samples were cooled down at a rate of $20^{\circ} \mathrm{C} / \mathrm{min}$ to $-80^{\circ} \mathrm{C}$ and then heated up to $20^{\circ} \mathrm{C}$ at a rate of $15^{\circ} \mathrm{C} / \mathrm{min}$. Glass transition $T_{\mathrm{g}}$ was determined by the DSC traces during the heating processes.

To understand the crystallization kinetics, all samples were subjected to fast scanning calorimetry (FSC) with Flash DSC (Mettler-Toledo Flash DSC 1) under the protection of nitrogen. The FSC sensor was conditioned and temperature-corrected according to the operating manual. The samples were microtomed into films, and the films were loaded on the 
sensor and melted therein to ensure good contact with the sensor. During the measurements, the samples were first annealed at $180^{\circ} \mathrm{C}$ to remove the thermal and flow history, cooled down at different rates, and then heated up at a fixed rate of $1000^{\circ} \mathrm{C} / \mathrm{s}$, during which the crystals, if formed during the first cooling process, would melt, allowing calculation of the degree of crystallization by the resulting enthalpy (area integrated on the melting peak). This protocol was repeated by increasing the cooling rate until the sample could be effectively quenched and no melting peak could be detected in the heating process.

\subsection{Conventional rheometry}

Linear viscoelasticity (LVE) measurements were conducted on the MCR-302 (Anton Paar) and ARES-G2 (TA Instrument) with $25 \mathrm{~mm}$ parallel plates. The samples were loaded at $180^{\circ} \mathrm{C}$ and annealed for $10 \mathrm{~min}$ to remove thermal history. After that, frequency sweep measurements were conducted every $10-20^{\circ} \mathrm{C}$ from $180^{\circ} \mathrm{C}$ to lower $T$ until the sample started to crystallize and $G^{\prime}$ increased with time at given $T$. During the frequency sweep measurements, strain amplitude was kept small $(\leq 10 \%)$ to ensure that the storage and loss moduli, $G^{\prime}$ and $G^{\prime \prime}$, were obtained within the linear regime as confirmed with the strain sweep measurements.

\subsection{Quartz resonator}

Our homemade quartz resonator is equipped with an AT-cut quartz crystal $(14 \mathrm{~mm}$ in diameter, Hangzhou Longqin Advanced Materials Sci \& Tech Co., Ltd., Hangzhou, China) 
with gold electrodes ( $5 \mathrm{~mm}$ in diameter) on both sides, for which the basic frequency is 6 $\mathrm{MHz}$, and could be extended to $3^{\text {rd }}, 5^{\text {th }}$ and $7^{\text {th }}$ overtones.

Before measuring the sample, the bare quartz crystal was loaded on a homemade holder, and the holder was placed into an oven (V0 200, Memmert GmbH). After a two-hour equilibration at each testing $T\left(=115.3,126.7,150.5\right.$ and $\left.160.0^{\circ} \mathrm{C}\right)$ in the oven, the resonance curve of bare quartz crystal was measured by using a quartz crystal microbalance based on admittance analysis (QCM-A DBY-17, Hangzhou Longqin Advanced Materials Sci \& Tech Co., Ltd., Hangzhou, China) with a control program, "QCM-DBY". By fitting the quartz crystal resonance curves, the resonance frequency $\omega_{0}$ and width at half-height $D_{0}$ of the resonance peak were determined at each basic frequency and overtone at each $T$. After that, a sample membrane with predetermined diameter $(\sim 5 \mathrm{~mm})$ and thickness $(0.3 \mathrm{~mm})$, prepared with a homemade compressor, was loaded on the gold electrode at one side of the quartz crystal. The sample and the quartz crystal were placed back into the oven and heated to $160.0^{\circ} \mathrm{C}$ in vacuum. This process ensured that sample and quartz crystal would maintain good contact.

Next, the quartz crystal coated with sample was heated at each testing $T$ in the oven for a two-hour equilibration. The resonance curve of the sample-coated quartz crystal was then measured again to give the resonance angular frequency $\omega$ and width at half-height $D$ of the resonance peak at each basic frequency and overtone. Moduli were determined from shift of angular frequency $\Delta \omega=\omega-\omega_{0}$ and change of peak width, as $\Delta D=D-D_{0}$ (both in unit of $\mathrm{rad} / \mathrm{s})$, in the following equations:

$$
\begin{aligned}
& G^{\prime}=k\left(\Delta D^{2} / 4-\Delta \omega^{2}\right) \\
& G^{\prime \prime}=-k \Delta D \Delta \omega
\end{aligned}
$$


Here, $k=\left(d_{\mathrm{q}} \rho_{\mathrm{q}}\right)^{2} / \rho_{\mathrm{p}}$ is a prefactor (Note that $k$ is different by a factor of $4 \pi^{2}$ from that reported in Ref [7] due to different units of frequency, i.e. $\mathrm{rad} / \mathrm{s}$ here and $\mathrm{Hz}$ in Ref [7].), $d_{\mathrm{q}}$ and $\rho_{\mathrm{q}}$ are the thickness and density of quartz, respectively, and $\rho_{\mathrm{p}}$ is the density of the probing sample. From Eq. 2(a) and (b), we see that $\tan \delta=G^{\prime \prime} / G^{\prime}=\Delta D \Delta \omega /\left(\Delta D^{2} / 4-\Delta \omega^{2}\right)$ is directly related to $\Delta D$ and $\Delta \omega$.

In the QR measurement, $G^{\prime}$ and $G^{\prime \prime}$ do not depend on membrane thickness as long as this membrane is sufficiently thick and fully covers one of the two gold electrodes. The penetration depth of acoustic shear wave generated by the quartz resonator in a viscoelastic liquid $p$ is given as $p=(2 \eta / \omega \rho)^{1 / 2}=\left(2 G / \omega^{2} \rho\right)^{1 / 2}$, where $\rho$ is the density of the viscoelastic liquid, and $\eta$ and $G$ are viscosity and shear modulus of the viscoelastic liquid, respectively. [12] The largest penetration depth $p$ of the shear wave can then be estimated to be about $37.5 \mu \mathrm{m}$ by taking $\rho$ of $1.0 \mathrm{~g} / \mathrm{cm}^{3}$ and $G$ of $1 \mathrm{GPa}$ as the fundamental frequency of the quartz resonator at $6 \mathrm{MHz}$, which is far smaller than the thickness of sample, i.e., $\sim 0.3 \mathrm{~mm}$. For higher $3^{\text {rd }}, 5^{\text {th }}$ and $7^{\text {th }}$ overtones, the penetration depths are even smaller. In such cases, the quartz resonator senses the only a thin layer of the bulk sample in melt state.

\section{Theory}

The modulus is expressed as a sum of the Rouse part, $G_{\mathrm{R}}$, and reptation part, $G_{\text {rep }}$, as

$$
G(t)=G_{\text {Rouse }}(t)+G_{\text {rep }}(t)
$$

The Rouse part can be written as 


$$
G_{\text {Rouse }}(t)=G_{\mathrm{e}} \sum_{N} \frac{N_{\mathrm{e}}}{N} \phi(N)\left\{\frac{1}{5} \sum_{q=1}^{N / N_{\mathrm{e}}} \exp \left(\frac{-t q^{2}}{\tau_{\mathrm{e}}\left(N / N_{\mathrm{e}}\right)^{2}}\right)+\sum_{N / N_{\mathrm{e}}+1}^{N} \exp \left(\frac{-t q^{2}}{\tau_{\mathrm{e}}\left(N / N_{\mathrm{e}}\right)^{2}}\right)\right\}
$$

Here, $G_{\text {Rouse }}$ contains two types of relaxation modes. The first term in the brace corresponds to low-order motional modes of length scales larger than the entanglement length, and the second term corresponds to the high-order motional modes corresponding to length scales smaller than the entanglement length. For the first term, a prefactor of $1 / 5$ is added, as derived by Likhtman and McLeish. [13]

The reptation part of modulus, on the other hand, can be expressed in the double reptation model [14-17] as

$$
G_{\text {rep }}(t)=G_{\mathrm{e}}\left(\sum_{N} \phi(N) F(t, N)\right)^{2}
$$

where a power 2 reasonably accounts for the constraint release mechanism. $F(t, N)$ is expressed in a form by

$$
F(t)=\frac{8}{\pi^{2}} \sum_{\text {odd } q} \frac{1}{q^{2}} \exp \left[-q^{2} U(t)\right]
$$

with $\quad U(t)=\frac{t}{\tau_{\text {rep }}}+\frac{\beta M_{\mathrm{e}}}{M} g\left[\frac{t M}{\tau_{\text {rep }} \beta M_{\mathrm{e}}}\right]$ and $g(x)=\sum_{m=1}^{\infty} \frac{1-\exp \left(-m^{2} x\right)}{m^{2}}$

\section{Results and discussion}

\subsection{Molecular weight distribution}


The static light scattering detector monitors the Rayleigh ratio $R_{\theta, \mathrm{i}}$ of the $i$-th fraction, which is related to the fraction concentration $C_{i}$ as

$$
\frac{K C_{i}}{R_{\theta, i}}=\frac{1}{P(\theta)_{i} M_{\mathrm{w}, i}}+2 A_{2, i} C_{i}+\ldots
$$

where the second and higher-order terms of $C_{i}$ can be ignored for dilute concentration employed in the GPC measurements. Rayleigh ratios at two angles of the TALLS detector, $\theta_{1}=15^{\circ}$ and $\theta_{2}=90^{\circ}$, enable calculation of their ratio $Z_{\mathrm{i}}=R \theta 1, \mathrm{i} / R \theta 2, \mathrm{i}=P\left(\theta_{1}\right)_{i} / P\left(\theta_{2}\right)_{i}$. Accordingly, the radius of gyration $R_{\mathrm{gi}}$ and the particle scattering function $P\left(\theta_{1}\right)_{\mathrm{i}}$ of the $i$-th fraction can be obtained from the ratio $Z_{\mathrm{i}}$ by the built-in Cirrus software with the random coil assumption for the particle scattering function. Then, the molecular weight $M_{\mathrm{i}}$ of the fraction is calculated with the known concentration from the DRI detector. [18; 19]

Figure 1 shows plots of $M$ as functions of the elution volume $V$ for all $\mathrm{PB}$ and $\mathrm{PBE}$ samples in this study. The difference among samples is trivial based on the similarity of their chemical structures. Scattering of data points is noted at both ends of the chromatograms since $M_{\mathrm{w}, \mathrm{i}}$ is, in principle, calculated from a ratio of the LS and RI signals that are proportional to $C_{\mathrm{i}} M_{\mathrm{w}, \mathrm{i}}$ and $C_{\mathrm{i}}$, respectively, and, thus, errors would appear when either of the signals approaches zero. To solve this data scattering problem, we use linear fit of the $\log M-$ $V$ plots at medium $V$ where the scattering is sufficiently small $\left(R^{2}\right.$ value $\left.\geq 0.997\right)$ and extrapolate the line to the high and low ends of elution volumes. This linear assumption of the calibration curves should hold for the samples in this study because the employed PLgel $10 \mu \mathrm{m}$ MIXED-B LS GPC columns have a linear molecular weight range of 500-10,000,000 $\mathrm{g} / \mathrm{mol}$. 


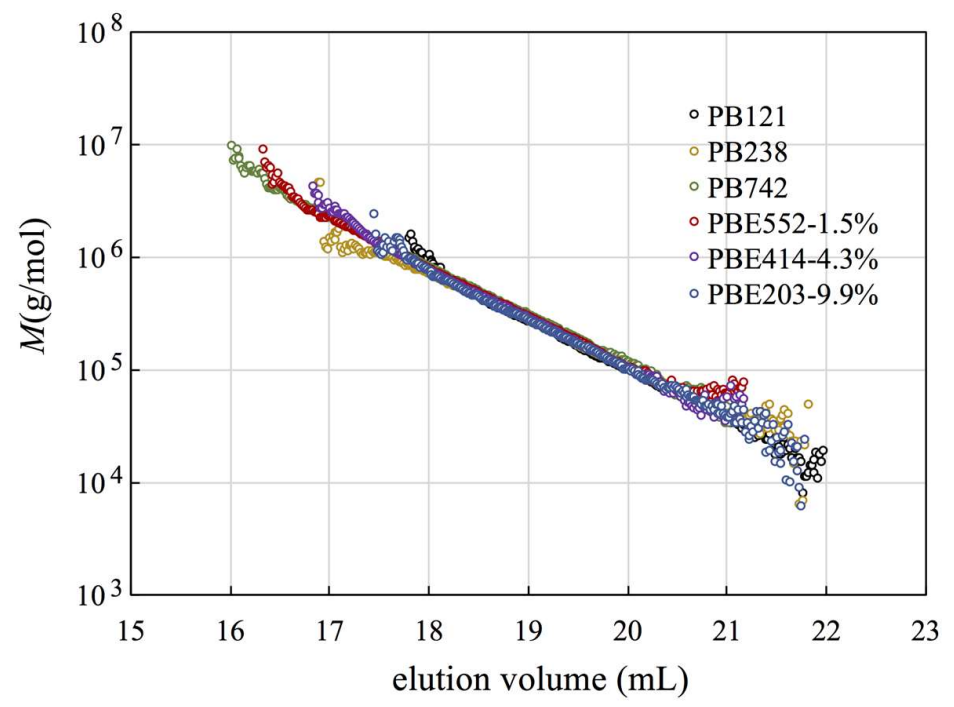

Figure 1: Molecular weight $M$ as a function of elution volume $V$ for all $\mathrm{PB}$ and PBE samples obtained by the multi-detector GPC.

With the absolute calibration curve of each sample established, the molecular weight distributions of the PB and PBE samples can be obtained, and the results are shown in Figure 2. The overall trend is a broadening of the molecular weight distribution with increasing molecular weight, as demonstrated in the plots and the $M_{\mathrm{w}} / M_{\mathrm{n}}$ values listed in Table 1 , which should be related to the detailed synthesis procedure that is unknown to us.

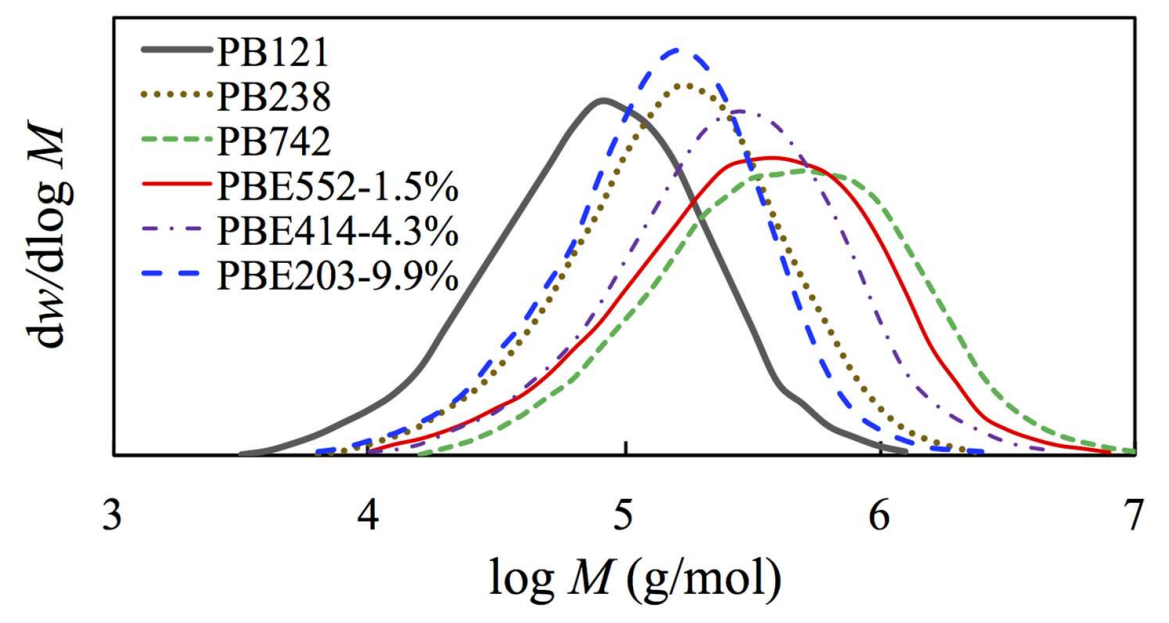

Figure 2: Molecular weight distributions of PB and PBE samples. 


\subsection{Linear viscoelasticity}

Figure 3 shows the storage and loss moduli, $G^{\prime}$ and $G^{\prime \prime}$, measured as functions of angular frequency $\omega$ for all $\mathrm{PB}$ and $\mathrm{PBE}$ samples reduced at a reference $T_{\mathrm{r}}=120^{\circ} \mathrm{C}$. The moduli are multiplied by an intensity factor, $b_{\mathrm{T}}=T_{\mathrm{r}} / T$, and shifted horizontally to construct the master curves. In principle, the modulus having entropic origin should be proportional to $\rho T$, and a normalization factor should be $\rho_{\mathrm{r}} T_{\mathrm{r}} / \rho T$. Nevertheless, a change of $\rho$ is usually much weaker than $T$ itself; therefore, we ignore the change of $\rho$ in our analysis. Time-temperature superposition can be achieved very well for the low $\omega b_{\mathrm{T}} G^{\prime}$ and $b_{\mathrm{T}} G^{\prime \prime}$ data measured from conventional rheometry, for which the uncertainty of each data point is usually less than \pm $5 \%$, corresponding to \pm 0.02 in the logarithmic scale, i.e., the size of symbols. In comparison, a similar quality of data cannot be achieved for the high $\omega b_{\mathrm{T}} G^{\prime}$ and $b_{\mathrm{T}} G^{\prime \prime}$ data detected in QR, which shows the scattering data quite clearly. For these data, it is difficult to shift the QR data precisely up to $\sim 0.02$ in logarithmic scale along the horizontal axis. Therefore, we shifted the data under the guidance of low $\omega$ LVE data by conventional rheometry. That is, we shifted the QR data detected at $115.3,126.7,150.5$ and $160.0^{\circ} \mathrm{C}$ by interpolating points of the plots of $a_{\mathrm{T}}$ against $T$ of conventional rheometry, assuming that high and low $\omega$ data exhibit the same temperature dependence. Since the temperature range of $\mathrm{QR}$ measurements is well within that of conventional rheological measurements, this protocol should be acceptable. The shift factors of LVE data by conventional rheometry and the interpolation points of QR are summarized in Figure 4 (a). The master curves thus constructed are further shifted by a vertical factor $\mathrm{A}$, as indicated (larger $\mathrm{A}$ is chosen for higher $M$ sample), to avoid overlapping. 
The solid curves in Figure 3 are predicted on the basis of the double reptation model by utilizing density $\rho=0.813 \mathrm{~g} / \mathrm{cm}^{3}$, as reported by Graessley. [20] The temperature for this reported density is $140^{\circ} \mathrm{C}$, very close to $T_{\mathrm{r}}=120^{\circ} \mathrm{C}$ in this study. The fitting parameters are summarized in Table 3 , including a fixed shape parameter $\beta$ (fixed to be $\beta=9$, identical to $\beta$ $=9$ reported for poly(vinyl ethylene) [21] and close to $\beta=8.7$ reported for polystyrene [15]), entanglement molecular weight $M_{\mathrm{e}}$ and Rouse time per Kuhn segment $\tau_{0}$. We first determined this factor $\beta$ through fitting LVE of pure PB and utilized the same factor for all the samples. Since a fixed $\beta$ enables us to achieve good predictions, we did not intend to introduce one additional adjustable parameter. The molecular weight per Kuhn segment is $M_{\mathrm{K}}=133 \mathrm{~g} / \mathrm{mol}$ for $\mathrm{PB}$, which is assumed to be the same for the PBE samples. This assumption is innocuous here since (1) a fraction of PE is low $\left(f_{\mathrm{PE}}<10 \%\right)$ and (2) all LVE studied here are below $G_{\mathrm{K}}$ $=\rho R T / M_{\mathrm{K}}=2 \times 10^{7} \mathrm{~Pa}$. Thus, while the choice of $M_{\mathrm{K}}$ would affect $\tau_{0}$, it would not affect predictive quality. In the model calculation, a power region $G^{\prime}=G_{\mathrm{K}}\left(\omega \tau_{0}\right)^{1 / 2}$ can be used to guide the choice of $\tau_{0}$. The molecular weight distribution $\phi(N)$ with $N=M / M_{0}$ in Eq. 5 was shown earlier in Figure 2. By utilizing $M_{\mathrm{e}}$ as a fitting paraemeter, we can calculate entanglement time $\tau_{\mathrm{e}}=\tau_{0}\left(M_{\mathrm{e}} / M_{0}\right)^{2}$ and reptation time $\tau_{\text {rep }}=\tau_{\mathrm{e}}\left(M / M_{\mathrm{e}}\right)^{3}$ in Eqs. 3-6. These parameters enable quantitative prediction of LVE in the frequency range of our measurements based on the double reptation model.

In Appendix B, we fit LVE based on the hierarchical model by Wang et al. by utilizing the same $M$ distribution and $\tau_{0}$, as shown in Figure 3, and $M_{\mathrm{e}}$ as the fitting parameters. [22] Good agreement could also be achieved between the experimental results and the theoretical prediction (cf. Figure B1). 
Since the calculations, as expressed in Eqs. 3-6, consider only the rubbery part of modulus, the predicted glassy-to-rubbery transition is characterized by $G^{\prime}=G^{\prime \prime} \sim \omega^{1 / 2}$ that ends at high $\omega$ where $G^{\prime}\left(=G_{\mathrm{K}}\right)$ becomes higher than $G^{\prime \prime}$. In contrast to this expectation, it is clear that $G^{\prime \prime}$ is higher than $G^{\prime}$ at the high $\omega$ end. This feature indicates that the glassy part of $G^{\prime \prime}$ starts to play a role at the high frequency end of the glassy-to-rubbery transition region, as observed extensively in the rheo-optical measurements performed by Inoue and coworkers. [23; 24] To address the contribution of glassy modulus, [23-25] we added $G_{\text {glassy" }}=\eta_{\text {glassy }} \omega$ to the rubbery part of $G^{\prime \prime}$, where $\eta_{\text {glassy }}=0.045 \mathrm{~Pa}$.s chosen to be the same for all the samples. This step leads to $G^{\prime \prime}>G^{\prime}$ at high frequency (see the dashed curves), which agrees with the experimental results. We should note that the glass modulus can be represented in more precise ways. For example, Marin and coworkers have suggested that the complex $G_{\text {glassy }}$ * can be represented as $G_{\text {glassy }}(\omega)=G_{\text {inf }}-G_{\text {inf }} /\left(1+i \omega \tau_{\text {glassy }}\right)^{1 / 2}$, or in time domain as $G_{\mathrm{HF}}(t)=$ $G_{\text {inf }}\left(1-\operatorname{erf}\left(t / \tau_{\text {glassy }}\right)^{1 / 2}\right) .[26 ; 27]$ An alternative representation is the Kohlrausch-William-Watts $(\mathrm{KWW})$ equation $\left.G_{\text {glassy }}(t)=G_{\text {infexp }}\left(-t / \tau_{\text {glassy }}\right)^{\beta}\right)$. [28-30] Since both the models above would give $G_{\text {glassy" }}=\eta_{\text {glassy }} \omega$ and $G_{\text {glassy }}{ }^{\prime}>G_{\text {glassy' }}$ in the glassy-to-rubbery transition region of current interest, we may safely use $G_{\text {glassy" }}=\eta_{\text {glassy }} \omega$ here as an approximation. 


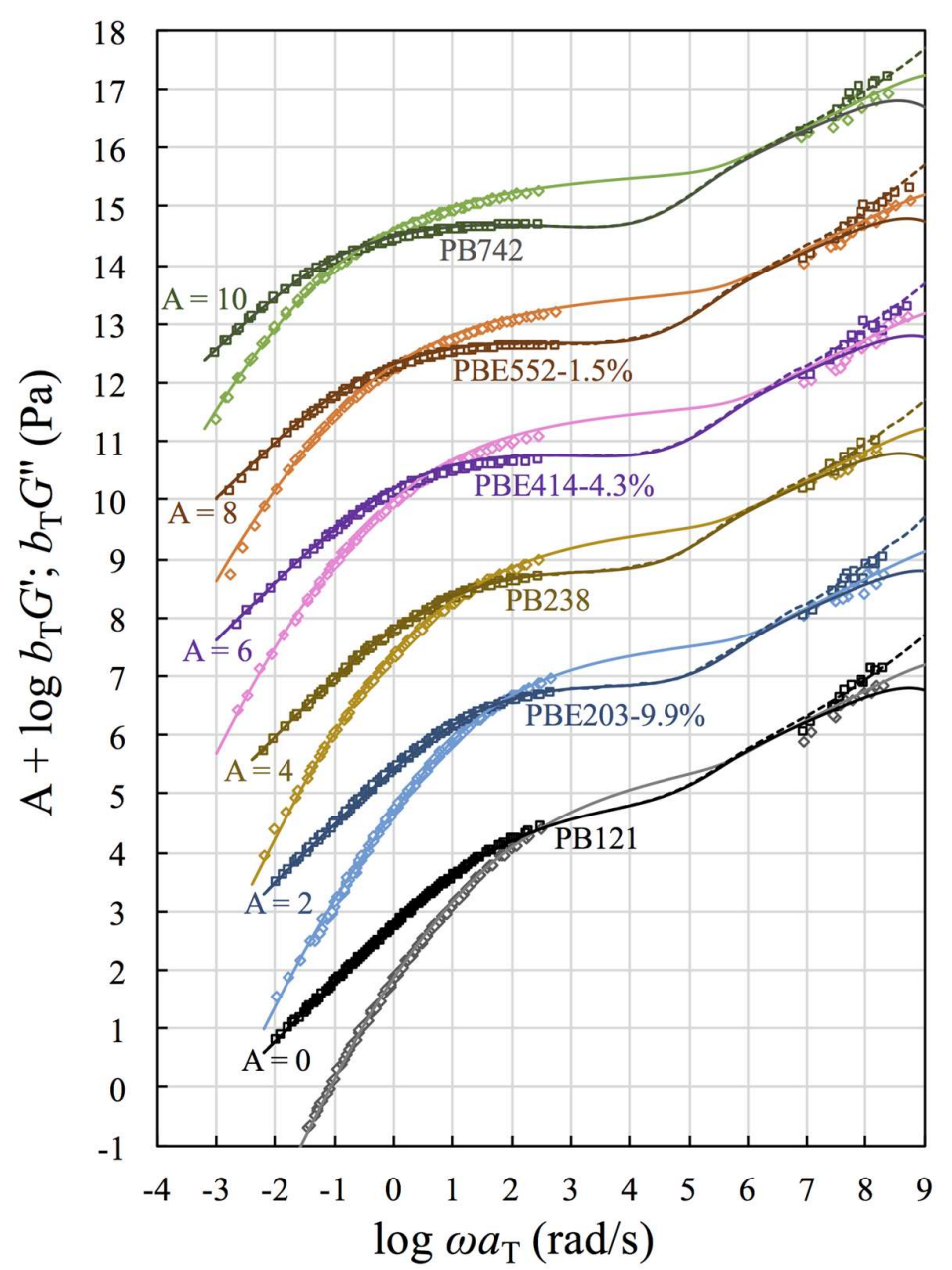

Figure 3: Comparison of LVE master curves (symbols) of storage and loss modulus, $G^{\prime}$ and $G^{\prime \prime}$, measured as a function of angular frequency $\omega$ and reduced at reference temperature $T_{\mathrm{r}}=$ $120^{\circ} \mathrm{C}$ for the PB and PBE samples and prediction (curves) of the double reptation model.

The LVE data are shifted along the vertical axis by a factor of $\mathrm{A}$ as indicated, where larger $\mathrm{A}$ was chosen for higher $M$ sample.

Table 3. Inputting parameters for model calculation at reference $T_{\mathrm{r}}=120^{\circ} \mathrm{C}$

$\begin{array}{lllll}\text { Brand Name } & \text { Code } & M_{\mathrm{e}}(\mathrm{g} / \mathrm{mol}) & \tau_{0}(\mathrm{~ns}) & \beta\end{array}$




\begin{tabular}{ccccc}
\hline PB0800 & PB121 & 7,500 & 0.66 & 9 \\
PB0400 & PB238 & 7,000 & 0.93 & 9 \\
PB0110 & PB742 & 7,500 & 1.0 & 9 \\
PB8640 & PBE552-1.5\% & 7,500 & 0.63 & 9 \\
PB8220 & PBE414-4.3\% & 6,700 & 0.59 & 9 \\
PB8510 & PBE203-9.9\% & 6,300 & 0.43 & 9 \\
\hline
\end{tabular}

\subsection{Effective glass transition temperature and segmental time}

The shift factors $a_{\mathrm{T}}$ of the PB and PBE samples are plotted against $T$ in Figure 4 (a). The temperature dependencies do not agree well, suggesting that $T_{\mathrm{g}}$ is different for these samples. Graessley and coworkers analyzed the viscoelastic shift factors of random PBE samples obtained through hydrogenating monodispersed polybutadiene samples of different ratios of 1,4 and 3,4 additions. They found that an empirical Williams-Landel-Ferry (WLF) equation holds for all PBE samples having various $\mathrm{PB}$ and $\mathrm{PE}$ ratios [4-6], as

$$
\log a_{\mathrm{T}}=\frac{-6.35\left(T-T_{g}-77\right)}{146+\left(T-T_{g}-77\right)}
$$

By choosing an iso-frictional temperature $T_{\text {iso }}=T_{\mathrm{g}}+77$, as the reference temperature, we can rewrite Eq. 8 as $\log a_{\mathrm{T}}=-6.35\left(T-T_{\text {iso }}\right) /\left(146+T-T_{\text {iso }}\right)$, as shown in curve in Figure $4(\mathrm{~b})$. By taking $T_{\text {iso }}$ as an adjustable parameter, we check the consistency between the plots of log $a_{\text {iso }}=\log a_{\mathrm{T}}\left(T_{\mathrm{r}}\right)-6.35\left(T_{\mathrm{r}}-T_{\text {iso }}\right) /\left(146+T_{\mathrm{r}}-T_{\text {iso }}\right)$ against $T-T_{\text {iso }}$ (symbols in Figure $\left.4(\mathrm{~b})\right)$ and the prediction by Eq. 8 (curve in Figure 4(b)) until the best agreement can be achieved. The scattering of data points is effectively reduced compared with those in Figure 4(a). From the 
fitting parameter $T_{\text {iso }}$ thus determined, we can further calculate the effective $T_{\mathrm{g}}$ as $T_{\mathrm{g}}=T_{\text {iso }}-$ 77, which is summarized in Table 1 and will be compared with the DSC $T_{\mathrm{g}}$ later in Figure 5.
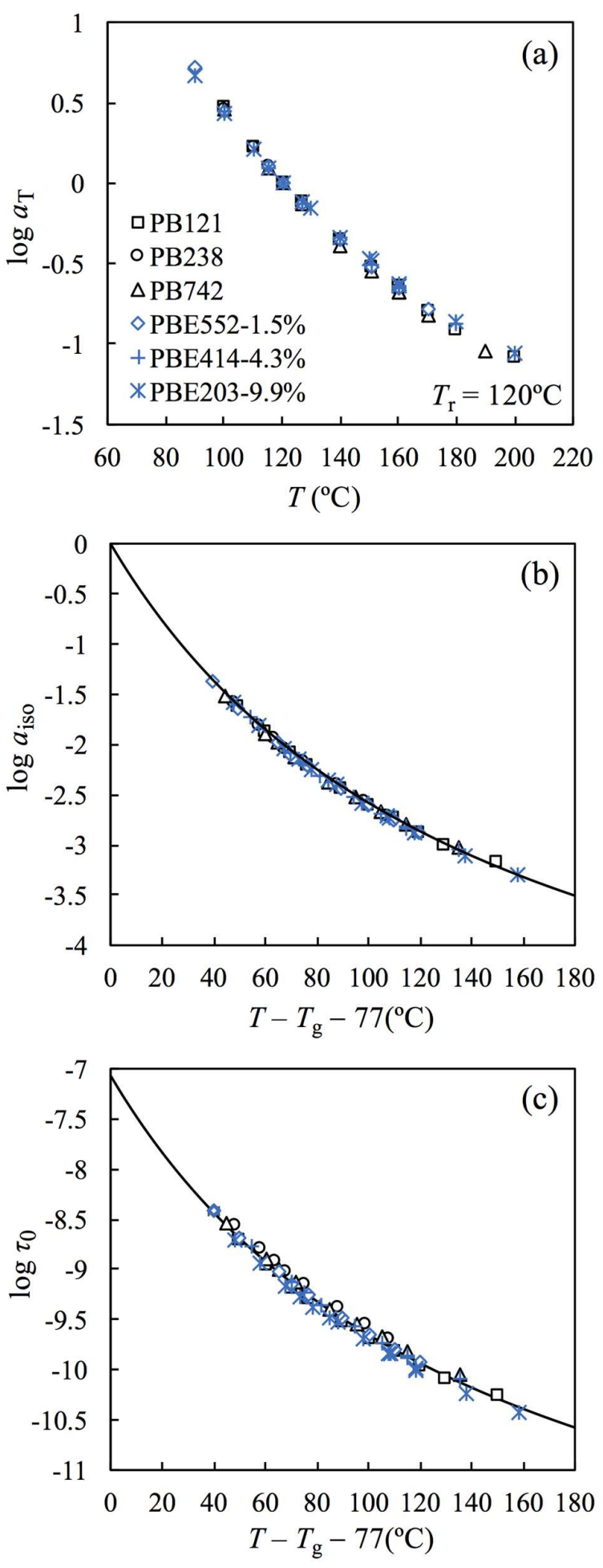
Figure 4: Comparison of (a) viscoelastic shift factor $a_{\mathrm{T}}$ against $T$ for the PB and PBE samples, with reference temperature $T_{\mathrm{r}}=120^{\circ} \mathrm{C}$, (b) viscoelastic shift factor $a_{\text {iso }}$ reduced to an isofrictional temperature $\left(T-T_{\mathrm{g}}-77\right)^{\circ} \mathrm{C}$ when $a_{\text {iso }}$ shows the same temperature dependence and can be fit by WLF Eq. 8, and (c) segmental time $\tau_{0}$ reduced to $\left(T-T_{\mathrm{g}}-77\right)^{\circ} \mathrm{C}$.

Since the samples are reduced at an iso-frictional state at $\left(T-T_{\mathrm{g}}-77\right){ }^{\circ} \mathrm{C}$ (Figure $\left.4(\mathrm{~b})\right)$, it can be expected that the segmental time can be properly normalized at $\left(T-T_{\mathrm{g}}-77\right){ }^{\circ} \mathrm{C}$. This expectation is tested in Figure 4 (c), showing that the segmental time $\tau_{0}$ extracted from the analysis of LVE at $T_{\mathrm{r}}$ (in Figure 3) is extended to another $T$ through the viscoelastic shift factor $a_{\mathrm{T}}$ and plotted against $\left(T-T_{\mathrm{g}}-77\right)^{\circ} \mathrm{C}$ (i.e., $T-T_{\text {iso }}$ ). It is remarkable that the plots of $\tau_{0}$ against $\left(T-T_{\mathrm{g}}-77\right)^{\circ} \mathrm{C}$ agree for all $\mathrm{PB}$ and PBE samples. The solid curve is defined by [4]

$$
\log \tau_{0}=-7.07-\frac{6.35\left(T-T_{\mathrm{g}}-77\right)}{146+T-T_{\mathrm{g}}-77}
$$

which is a reasonable representation of $\tau_{0}$ obtained for all the PB and PBE samples in this study, as $\log \tau_{0}=-7.07$ at $T=\left(T_{\mathrm{g}}+77\right)^{\circ} \mathrm{C}$.

Figure 5 compares $T_{\mathrm{g}}$ plotted against the fraction of PE from our WLF analysis (filled diamonds) and that by Graessley and coworkers (filled spheres), both representing $T_{\mathrm{g}}$ of the samples in melt, [4] and $T_{\mathrm{g}}$ measured directly from DSC by us (unfilled diamonds) and by De Rosa et al. (unfilled squares), both representing $T_{\mathrm{g}}$ of the semi-crystallized samples. [31] It is worth mentioning that the samples studied by De Rosa et al. are stereo-regular samples synthesized with the zirconocene catalyst. Difference is revealed from comparing $T_{\mathrm{g}}$ from the WLF analysis (filled diamonds) and that from the DSC measurements (unfilled diamonds) in this study, which is not surprising because the former is given on the melt samples while the latter is on the amorphous phase of the semi-crystallized samples. This difference becomes 
smaller for the PBE203-9.9\% sample possibly because it has the lowest degree of crystallization owing to the highest content of PE. Although the difference exists, the overall trend is similar in that $T_{\mathrm{g}}$ decreases with increasing fraction of ethylene $\left(f_{\mathrm{PE}}\right)$ comonomers.

The dashed curve corresponds to the prediction of the empirical Fox equation: [32]

$$
T_{g}=\frac{f_{\mathrm{PE}}}{T_{\mathrm{g}, \mathrm{PE}}}+\frac{1-f_{\mathrm{PE}}}{T_{\mathrm{g}, \mathrm{PB}}}
$$

which underestimates the plasticizing effect of PE. In comparison, the empirical DiMarzio equation with one additional fitting parameter, $r=0.81$, can give better prediction: [33]

$$
T_{g}=\frac{f_{\mathrm{PE}} T_{\mathrm{g}, \mathrm{PE}}+r\left(1-f_{\mathrm{PE}}\right) T_{\mathrm{g}, \mathrm{PB}}}{f_{\mathrm{PE}}+r\left(1-f_{\mathrm{PE}}\right)}
$$

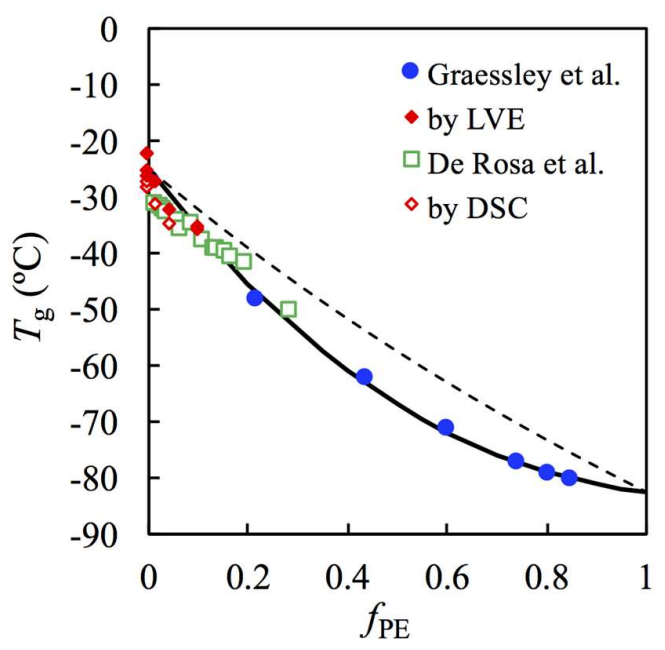

Figure 5: Comparison of effective glass transition temperature $T_{\mathrm{g}}$ against a number fraction of ethylene monomers. The filled spherical symbols are data reported by Graessley and coworkers, and the unfilled square symbols are data of De Rosa et al, and filled and unfilled diamond symbols are data in this study. The solid and dashed curves correspond to predictions using the DiMarzio and Fox equations, respectively. 


\subsection{Entanglement molecular weight}

Figure 6 compares the entanglement molecular weights $\left(M_{\mathrm{e}}\right)$ of PB and PBE samples obtained from fitting the data to the double reptation model (square symbols) and the hierarchical model of Larson and coworkers (diamond symbols, cf. Appendix B for details), as well as that calculated from $G_{\mathrm{e}}$ reported by Graessley and coworkers (spherical symbols). [4] The overall trend is a decrease of $M_{\mathrm{e}}$ with increasing fraction of PE, reflecting higher chain flexibility for samples with higher $f_{\mathrm{PE}}$. The entanglement spacing of isotactic $\mathrm{PB}$ in this study is smaller than that of the atactic PB sample studied by Graessley and coworkers, as discussed below. [4]

One of the simplest rules that correlates the molecular weight per backbone and $M_{\mathrm{e}}$ is the "backbone equivalence" model. In this model, the size of a coil is assumed to be determined by the backbone PE so as to give the packing length $p \sim m_{\mathrm{b}}$. Since the entanglement length is proportional to the packing length as $a=P_{\mathrm{e}} p$, with $P_{\mathrm{e}} \sim 20$ appearing to be a constant, the volume per entanglement segment becomes $M_{\mathrm{e}} / \rho=a^{2} p=P_{\mathrm{e}}{ }^{2} p^{3} \sim m_{\mathrm{b}}{ }^{3}$. A difference of the density $\rho$ of different polyolefins could be ignored to give $[8 ; 34]$

$$
M_{\mathrm{e}}=M_{\mathrm{e}, \mathrm{PE}}\left(m_{\mathrm{b}} / m_{\mathrm{b}, \mathrm{PE}}\right)^{3}
$$

where $M_{\mathrm{e}, \mathrm{PE}}=1,000 \mathrm{~g} / \mathrm{mol}$ and $m_{\mathrm{b}, \mathrm{PE}}=14 \mathrm{~g} / \mathrm{mol}$ are the molecular weights per entanglement and per backbone of PE, respectively. This equation was derived earlier by Fetters, Lohse and coworkers (c.f. Eq. 13d of ref [34]). The backbone equivalence assumption can be interpreted as a constant ratio between trans/gauche conformational isomers (conformers). However, it is well known that this ratio changes according to the length and arrangements of 
the side chains, i.e., monomer type and the tacticity. Taking these factors into consideration, the simplest adaptation would use the power in Eq. 12 as a fitting parameter to give

$$
M_{\mathrm{e}}=M_{\mathrm{e}, \mathrm{PE}}\left(m_{\mathrm{b}} / m_{\mathrm{b}, \mathrm{PE}}\right)^{\alpha}
$$

where $\alpha$ reflects the roles of monomer chemistry and tacticity. Figure 6 compares $M_{\mathrm{e}}$ obtained for the heterotactic PB and PBE copolymers studied by Graessley and coworkers (blue spheres) and $M_{\mathrm{e}}$ obtained through fitting with the double reptation model (black squares) and hierarchical model (green diamonds). The solid and dashed lines correspond to the predictions of Eq. 13 with $\alpha=3.3$ and 4.2, respectively. The different $\alpha$ values suggest the important role played by tacticity.

Fetters, Lohse and coworkers compared a prediction of Eq. 13 with experimental results of more than ten different types of polyolefins and concluded that the plateau modulus $G_{\mathrm{N}} \sim m_{\mathrm{b}}{ }^{-}$ ${ }^{3.49}$ for $m_{\mathrm{b}}=14-28 \mathrm{~g} / \mathrm{mol}$ and $G_{\mathrm{N}} \sim m_{\mathrm{b}}{ }^{-1.58}$ for $m_{\mathrm{b}}=35-56 \mathrm{~g} / \mathrm{mol}$, corresponding to $M_{\mathrm{e}} \sim m_{\mathrm{b}}{ }^{3.49}$ (for $m_{\mathrm{b}}=14-28 \mathrm{~g} / \mathrm{mol}$ ) and $M_{\mathrm{e}} \sim m_{\mathrm{b}}{ }^{1.58}$ (for $m_{\mathrm{b}}=35-56 \mathrm{~g} / \mathrm{mol}$ ) if the difference of $\rho$ is negligible. [34] Meanwhile, they observed $\left\langle R^{2}>/ M \sim m_{\mathrm{b}}{ }^{-1.43}\right.$ at $298 \mathrm{~K}$ and $<R^{2}>/ M \sim m_{\mathrm{b}}{ }^{-1.30}$ at $373 / 463 \mathrm{~K}$ for $m_{\mathrm{b}}=14-28 \mathrm{~g} / \mathrm{mol}$, both stronger than $<R^{2}>/ M \sim m_{\mathrm{b}}{ }^{-1}$ expected from the backbone equivalence model, which they attributed to an enhanced ratio of gauche conformers with $m_{\mathrm{b}}$ by the presence of branching groups. The higher $M_{\mathrm{e}} \sim m_{\mathrm{b}}{ }^{3.49}$ compared to $M_{\mathrm{e}} \sim m_{\mathrm{b}}{ }^{3}$, as predicted by the backbone equivalence model, should somehow be related to this enhancement. The solid and dashed curves in Figure 6 represent the prediction of Eq. 13 with $\alpha=3.3$ and 4.1 , respectively, where the former value is close to 3.49 reported by Fetters, Lohse and coworkers.

The solid curve with $\alpha=3.3$ gives $M_{\mathrm{e}}=8,000$ for $f_{\mathrm{PE}}=0$, much smaller than $M_{\mathrm{e}}=13,500$, as determined from $G_{\mathrm{e}}$ reported by Graessley and coworkers. We suspect that this difference 
results from a difference in stereo-regularity. For the isotactic $\mathrm{PB}$ and $\mathrm{PBE}$ samples in this study, a ratio between trans and gauche conformers should be more similar to that of PE, giving a value of $\alpha=3.3$ close to a factor of 3 (that assumes this ratio to be unchanged). In contrast, for the heterotactic PB and PBE studied by Graessley and coworkers, the deviation of $\alpha$ becomes more prominent with increasing fraction of PB where $\alpha=4.1$ (dashed curve) is required to predict the behavior of the pure PB sample, possibly related to a lack of tacticity that leads to a change of the trans/gauche ratio.

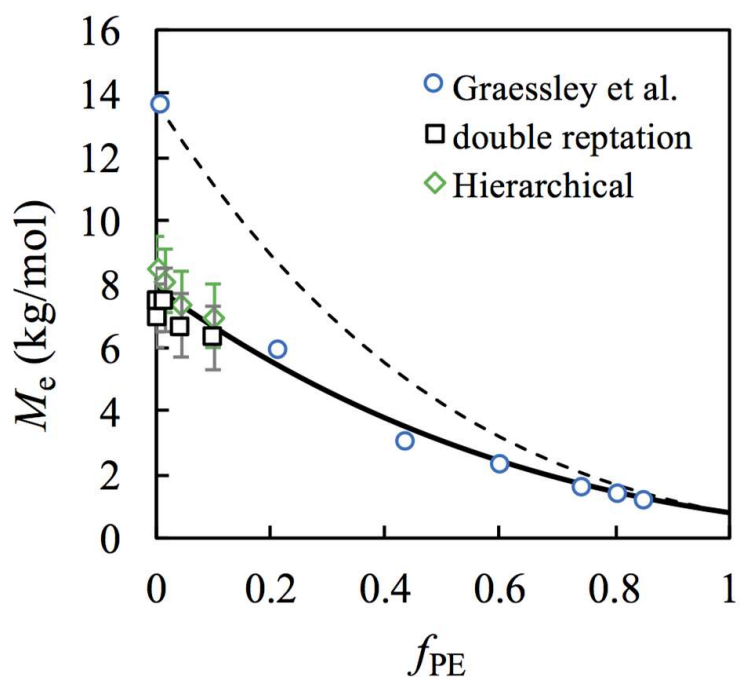

Figure 6: Comparison of $M_{\mathrm{e}}$ plotted against a number fraction of ethylene monomers of the PB and PBE samples. An error bar of \pm 1000 is estimated for $M_{\mathrm{e}}$ from data fitting. The spherical symbols are calculated from $G_{\mathrm{e}}$ reported by Graessley and coworkers, and the square and diamond symbols are obtained through fitting with the double reptation and hierarchical models, respectively. The solid and dash curves correspond to the prediction of Eq. 13 with $\alpha=3.3$ and 4.1, respectively.

\section{Conclusion}


This study explored the possibility of measuring the linear viscoelasticity of isotatic PB and PBE by using conventional rheometry in combination with the quartz resonator. The protocol of experiment and data analysis should be applicable to all semicrystalline polyolefins in melt.

The linear viscoelasticity from the Rouse to terminal relaxation could be detected above the crystallization temperature, which was further analyzed with the double reptation model. Such analysis enabled us to determine glass transition temperature $T_{\mathrm{g}}$ and entanglement molecular weight $\left(M_{\mathrm{e}}\right)$ of these samples.

Comparison of $T_{\mathrm{g}}$ and $M_{\mathrm{e}}$ with those parameters for atactic PB and PBE, studied by Graessley and coworkers, revealed the effects of stereo-regularity. The dependence of $T_{\mathrm{g}}$ on PE content seems to be consistent between isotactic and atactic samples. However, $M_{\mathrm{e}}$ is considerably larger for the atactic sample than the isotactic sample, suggesting the important role that stereo-regularity plays in chain flexibility.

Appendix A: Thermal analysis

Thermal measurements were conducted with both conventional DSC equipment Q20 (TA Instruments) and Flash DSC (METTLER TOLEDO Flash DSC 1). Conventional DSC equipment was used to characterize the glass transition temperature $T_{\mathrm{g}}$ of the semicrystallized samples, to be compared with that reported by De Rosa et al. on isotactic PBE copolymers synthesized with the zirconocene catalyst, [31] while Flash DSC was employed to understand the minimum cooling rate required to effectively quench the samples without crystallization. Understanding this criterion helps in evaluating if the LVE of these samples 
can be characterized through quenching the amorphous sample to a testing temperature without crystallization, a method that has been suggested by Kapnistos and Vlassopoulos. [35]

For the conventional DSC measurements, the samples were first annealed at $180^{\circ} \mathrm{C}$ for $10 \mathrm{~min}$ to remove the thermal and flow histories and then subjected to isothermal crystallization. After that, the samples were cooled down at a rate of $20^{\circ} \mathrm{C} / \mathrm{min}$ to $-80^{\circ} \mathrm{C}$ and then heated to $20^{\circ} \mathrm{C}$ at a rate of $15^{\circ} \mathrm{C} / \mathrm{min}$, during which the DSC traces were recorded and shown in Figure A1. The DSC traces of PB238, PB742, PBE552-1.5\%, PBE414-4.3\%, and PBE203-9.9\% were reported in our previous study, while the DCS trace of PB121 was only reported in this study. [36]

In Figure A1, the glass transition region becomes more prominent with increasing PE content because the introduction of ethylene reduces stereo-regularity and suppresses crystallization, leading to larger fraction of the amorphous phase that activates the stronger glass transition process. The glass transition temperature is chosen as the mid-point of the glass transition process, which increases slightly with increasing molecular weight owing to the well-known high free volume contribution of the chain ends, but decreases with increasing PE content, owing to the plasticizing effect of PE, which is similar to the trend reported in literature. [4; 37] 


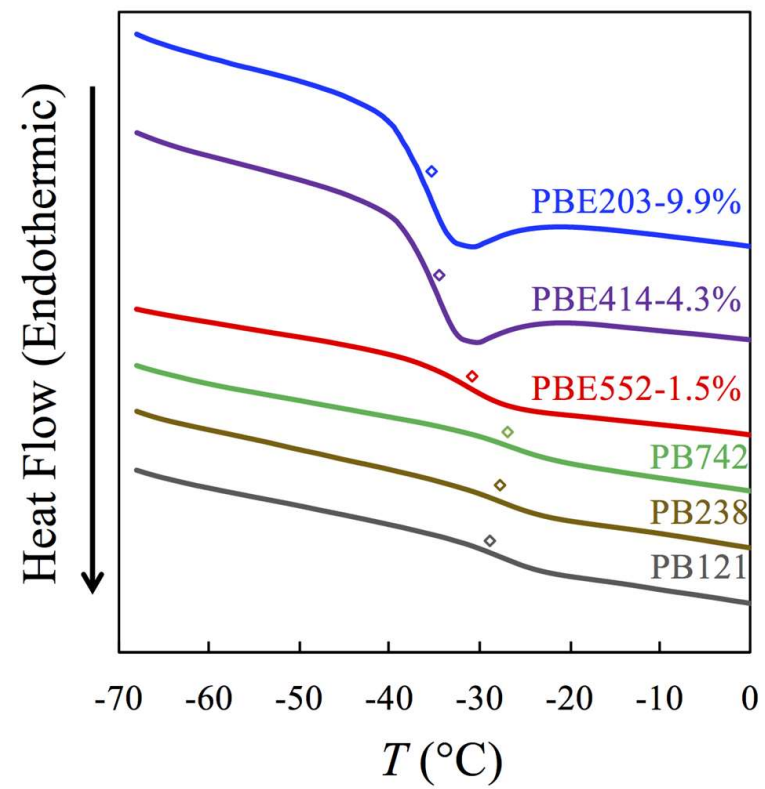

Figure A1: DSC traces of PB and PBE samples obtained after isothermal crystallization at different $T_{\mathrm{c}}$.

Figure A2 shows the fast scanning calorimetry (FSC) traces detected at a heating rate of $1000^{\circ} \mathrm{C} / \mathrm{s}$ after cooling down at different rates, as indicated. It can be seen that the threshold quenching rate to prevent crystallization is very high for the pure PB samples (top panels), i.e., $30^{\circ} \mathrm{C} / \mathrm{s}$ for the $\mathrm{PB} 121$ and $\mathrm{PB} 238$ samples and $100^{\circ} \mathrm{C} / \mathrm{s}$ for the $\mathrm{PB} 742$ sample. The threshold quenching rate gradually decreases for samples with higher content of ethylene comonomers by the suppression of crystallization kinetics after reducing stereo-regularity. From the bottom panels of Figure A2, we see that the threshold rates are $5^{\circ} \mathrm{C} / \mathrm{s}$ for the PBE552-1.5\% sample and $0.5^{\circ} \mathrm{C} / \mathrm{s}\left(30^{\circ} \mathrm{C} / \mathrm{min}\right)$ for the PBE414-4.3\% and PBE203-9.9\% samples. The latter value is close to the limited quenching rate of conventional rheometers. In addition, the quenching efficiency could be lower for the rheometers compared with the Flash DSC on the thinner sample membrane; therefore, we did not attempt to measure LVE of the effectively quenched samples. 


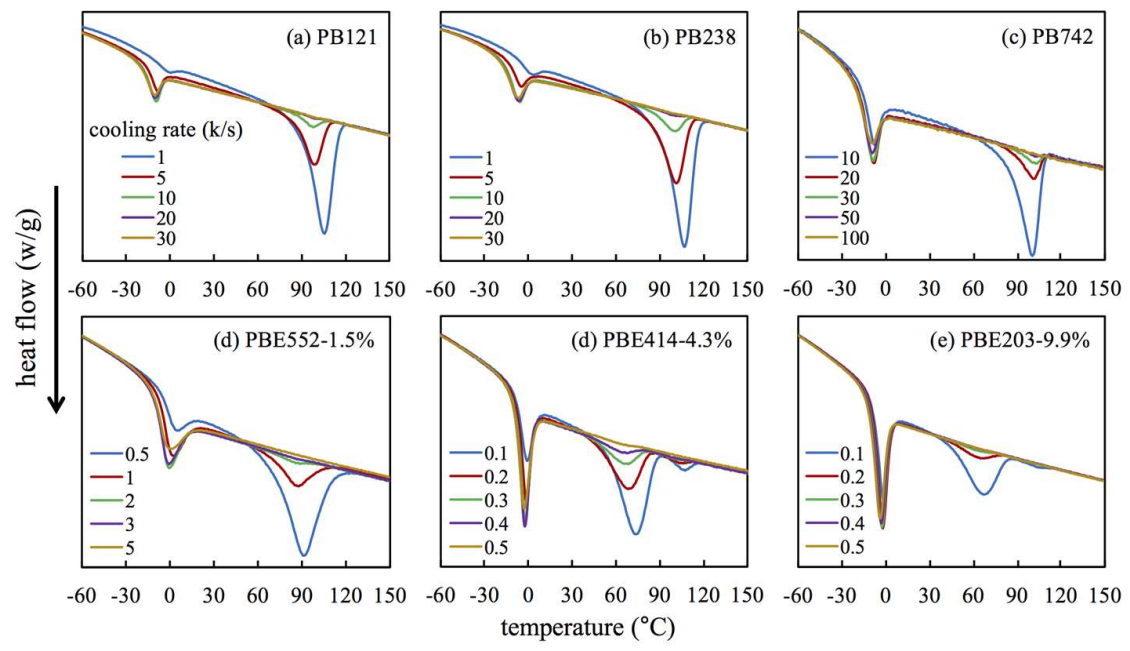

Figure A2: FSC traces recorded during heating at a rate of $1000^{\circ} \mathrm{C} / \mathrm{s}$ after quenching the $\mathrm{PB}$ and PBE samples at different rates, as indicated.

Appendix B: Prediction of Hierarchical Model

We compared the experimental results with theoretical predictions made by using the Hierarchical-3.0 Model [22]. The hierarchical model is a tube-based computational model originally developed by Larson (Hierarchical-1.0) [38] for predicting the linear viscoelasticity (LVE) of general mixtures of entangled linear and branched polymers. This model was later refined by Park et al. (Hierarchical-2.0) [39] and Wang et al. (Hierarchical-3.0), "which incorporates several refinements of the relaxation mechanisms and also shows a higher computational efficiency due to the use of a logarithmic time stepping method for calculating the time evolution of the arm-end retraction", as explained in the context of the present work below (Wang et al., 2010). [22] The general theoretical framework of the hierarchical model 
is based on the Milner-McLeish theory for blends of monodisperse star and linear polymers [40-42]

In the Milner-McLeish theories [40; 41] and, hence, the hierarchical model, entangled linear polymers are treated as symmetric two-arm stars with the arm-free ends retracting along the confining tubes of the polymers owing to contour length fluctuations until reptation takes over. For polydisperse polymer systems, constraint release (CR) effects are addressed by the dynamic tube dilation (DTD) theory. [43; 44] In addition, upon a sudden drop in the volume fraction of unrelaxed materials $(\Phi(t))$ in the system such that the DTD picture fails, relaxation enters a CR-Rouse regime whereby arm retraction occurs in a partially dilated tube, and the tube itself undergoes Rouse motion inside a wider supertube $[41 ; 42 ; 45]$. In the current work, we assume that the diameter of the partially dilated tube is determined by the unrelaxed volume fraction just before the onset time of the CR-Rouse regime, which is termed as the "thin" tube approach $[22 ; 46]$. The diameter of the supertube is determined by a supertube volume fraction which decreases with time in a power law, as $\Phi_{S T}(t) \sim t^{-1 / 2 \vartheta}$, where $\vartheta$ is the dilution exponent. The CR-Rouse regime ends when $\Phi_{S T}(t)$ reaches the unrelaxed volume fraction $\Phi(t)$. In a highly polydisperse system, the process of entering and exiting the CR-Rouse regime may take place many times before the stress relaxation of the entire system is terminated by the reptation of the longest chains.

The influence of different relaxation mechanisms, numerical implementation methods, and choice of certain model parameters on the LVE predictions of the hierarchical model and another tube-based computational model, the "bob" (or branch-on-branch) model [46], has been discussed in detail in a previous publication [22]. For the entangled linear polymer systems studied in this work, the only variable model parameter is the dilution exponent $\vartheta$ which can be chosen as either 1 or $4 / 3$. It has been shown in theoretical $[47 ; 48]$ and 
hierarchical model [22] calculations that the use of $\vartheta=1$ can provide better predictions for the viscoelastic properties of binary blends of linear polymers than $\vartheta=4 / 3$, which, however, works better for star-linear blends. The same trend has been found for the description of polydisperse linear PB and PBE melts. Therefore, all hierarchical model predictions presented here are generated using $\vartheta=1$.

The input system parameters for the hierarchical model calculations include the plateau modulus

$$
G_{N}^{0}=G_{e}=\frac{\rho R T}{M_{e}}
$$

and the equilibration or entanglement time

$$
\tau_{e}=\tau_{0}\left(\frac{M_{e}}{M_{0}}\right)^{2}
$$

where the same density and molar weight of monomers, $\rho=0.813 \mathrm{~g} / \mathrm{cm}^{3}$ and $M_{0}\left(=M_{\mathrm{k}}\right)=$ $133 \mathrm{~g} / \mathrm{mol}$, are used for all studied samples. The reference temperature is set as $T=120^{\circ} \mathrm{C}$. Both $M_{\mathrm{e}}$ and $\tau_{0}$ for different $\mathrm{PB}$ and PBE samples are summarized in Table B1. The experimentally determined molecular weight distributions, as shown in Figure 2, are also used directly in the model calculations.

In Figure B1, the hierarchical model predictions for the LVE of different $\mathrm{PB}$ and PBE samples are shown to be in reasonably good agreement with the experimental data. The slightly higher theoretical values in the terminal relaxation regime (lowest frequencies) can be attributed to the artificially extended CR-Rouse regime whereby arm retraction in the "thin" tube leads to a faster decay of the unrelaxed volume fraction $\Phi$ compared to that of supertube volume fraction $\Phi_{S T}$ and, as such, results in an overestimation of the stress moduli [22]. This problem is most severe in describing star-linear blends with very low volume fractions of star polymers, but relatively innocuous for the polydisperse linear polymer systems studied here, 
though still noticeable. The overestimation problem could be partly resolved by taking into account the disentanglement relaxation mechanism, which effectively cuts off the artificially extended CR-Rouse regime. [22; 38; 39]

Table B1. Inputting parameters for hierarchical model calculation at reference temperature $T_{\mathrm{r}}$ $=120^{\circ} \mathrm{C}$

\begin{tabular}{cccc}
\hline Brand Name & Code & $M_{\mathrm{e}}(\mathrm{g} / \mathrm{mol})$ & $\tau_{0}(\mathrm{~ns})$ \\
\hline PB0800 & PB121 & 7,500 & 0.66 \\
PB0400 & PB238 & 7,500 & 0.93 \\
PB0110 & PB742 & 8,500 & 1.0 \\
PB8640 & PBE552-1.5\% & 8,100 & 0.63 \\
PB8220 & PBE414-4.3\% & 7,400 & 0.59 \\
PB8510 & PBE203-9.9\% & 7,000 & 0.43 \\
\hline
\end{tabular}




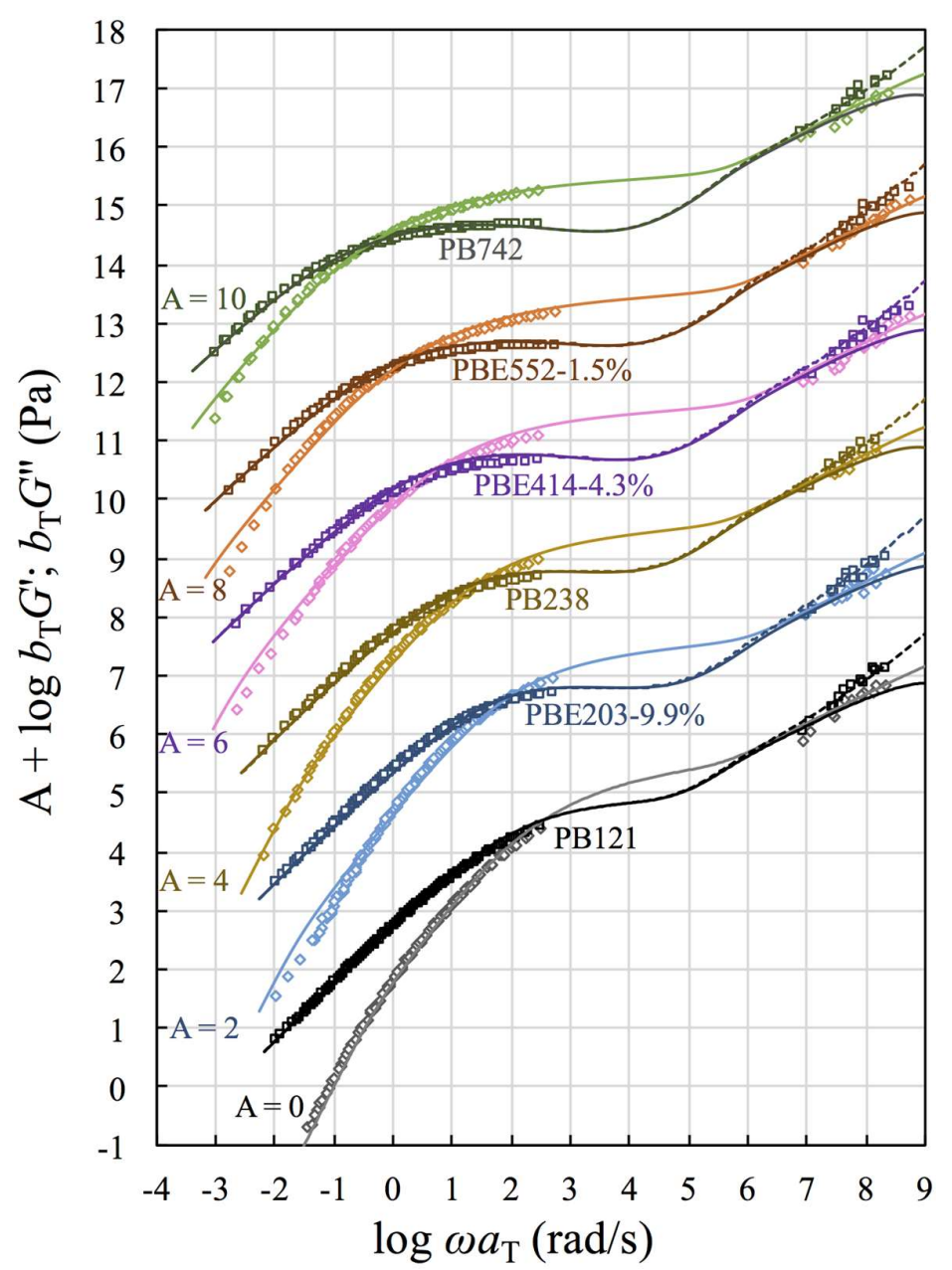

Figure B1: Comparison of LVE master curves (symbols) of storage and modulus, $G^{\prime}$ and $G^{\prime \prime}$, measured as a function of angular frequency $\omega$ and reduced at reference temperature $T_{\mathrm{r}}=$ $120^{\circ} \mathrm{C}$ for the PB and PBE samples and prediction of the hierarchical model. The LVE data are shifted along the vertical axis by a factor of A, as indicated, where larger A was chosen for higher $M$ sample.

Acknowledgements: 
Q.C. acknowledges the Natural Science Foundation of China (No. 21790343) and the Jilin

Scientific and Technological Development Program (Grant No. 20180519001JH). We thank

Baixiang Li for help with the GPC measurements, Professor Yanhu Xue for help with the ${ }^{13} \mathrm{C}$

NMR measurements, and Professor Yonggang Liu for insightful discussion regarding the GPC results.

\section{References}

[1] Acierno, S., B. Palomba, H. H. Winter and N. Grizzuti, "Effect of molecular weight on the flow-induced crystallization of isotactic poly(1-butene)," Rheol Acta 42, 243-250 (2003).

[2] Hadinata, C., C. Gabriel, M. Ruellmann, N. Kao and H. M. Laun, "Shear-induced crystallization of PB-1 up to processing-relevant shear rates," Rheol Acta 45, 539-546 (2006).

[3] Hadinata, C., C. Gabriel, M. Ruellmann, N. Kao and H. M. Laun, "Correlation between the gel time and quiescent/quasi-quiescent crystallization onset time of poly(butene1) as determined from rheological methods," Rheol Acta 45, 631-639 (2006).

[4] Carella, J. M., W. W. Graessley and L. J. Fetters, "Effects of chain microstructure on the viscoelastic properties of linear polymer melts: polybutadienes and hydrogenated polybutadienes," Macromolecules 17, 2775-2786 (1984).

[5] Fetters, L. J., W. W. Graessley, R. Krishnamoorti and D. J. Lohse, "Melt Chain Dimensions of Poly(ethylene-1-butene) Copolymers via Small Angle Neutron Scattering," Macromolecules 30, 4973-4977 (1997).

[6] Pearson, D. S., L. J. Fetters, W. W. Graessley, G. Ver Strate and E. von Meerwall, "Viscosity and self-diffusion coefficient of hydrogenated polybutadiene," Macromolecules 27, 711-719 (1994).

[7] Szanto, L., R. Vogt, J. Meier, D. Auhl, E. Van Ruymbeke and C. Friedrich, "Entanglement relaxation time of polyethylene melts from high-frequency rheometry in the mega-hertz range)," J Rheol 61, 1023-1033 (2017).

[8] Garcia-Franco, C. A., B. A. Harrington and D. J. Lohse, "Effect of short-chain branching on the rheology of polyolefins," Macromolecules 39, 2710-2717 (2006).

[9] Gahleitner, M., "Melt rheology of polyolefins," Progress in Polymer Science 26, 895944 (2001).

[10] Stolte, I. and R. Androsch, "Kinetics of the melt - Form II phase transition in isotactic random butene-1/ethylene copolymers," Polymer 54, 7033-7040 (2013). 
[11] Wang, Y. T., Y. Lu, J. Y. Zhao, Z. Y. Jiang and Y. F. Men, "Direct Formation of Different Crystalline Forms in Butene-1/Ethylene Copolymer via Manipulating Melt Temperature," Macromolecules 47, 8653-8662 (2014).

[12] Du, B. Y., E. Goubaidoulline and D. Johannsmann, "Effects of laterally heterogeneous slip on the resonance properties of quartz crystals immersed in liquids," Langmuir 20, 10617-10624 (2004).

[13] Likhtman, A. E. and T. C. B. McLeish, "Quantitative theory for linear dynamics of linear entangled polymers," Macromolecules 35, 6332-6343 (2002).

[14] Cloizeaux, J. D., "Relaxation and Viscosity Anomaly of Melts Made of Long Entangled Polymers - Time-Dependent Reptation," Macromolecules 23, 4678-4687 (1990).

[15] van Ruymbeke, E., R. Keunings, V. Stephenne, A. Hagenaars and C. Bailly, "Evaluation of reptation models for predicting the linear viscoelastic properties of entangled linear polymers," Macromolecules 35, 2689-2699 (2002).

[16] Chen, Q., Z. J. Zhang and R. H. Colby, "Viscoelasticity of entangled random polystyrene ionomers," J Rheol 60, 1031-1040 (2016).

[17] Pattamaprom, C. and R. G. Larson, "Predicting the linear viscoelastic properties of monodisperse and polydisperse polystyrenes and polyethylenes," Rheol Acta 40, 516-532 (2001).

[18] Mourey, T. H. and H. Coll, "Size-Exclusion Chromatography with 2-Angle Laser LightScattering (Sec-Talls) of High-Molecular-Weight and Branched Polymers," J Appl Polym Sci 56, 65-72 (1995).

[19] Liu, Y. G., S. Q. Bo, Y. J. Zhu and W. H. Zhang, "Determination of molecular weight and molecular sizes of polymers by high temperature gel permeation chromatography with a static and dynamic laser light scattering detector," Polymer 44, 7209-7220 (2003).

[20] Graessley, W. W., Polymeric Liquids and Networks : Dynamics and Rheology. (Garland Science, London ; New York, 2008).

[21] Pathak, J. A., S. K. Kumar and R. H. Colby, "Miscible polymer blend dynamics: Double reptation predictions of linear viscoelasticity in model blends of polyisoprene and poly(vinyl ethylene)," Macromolecules 37, 6994-7000 (2004).

[22] Wang, Z. W., X. Chen and R. G. Larson, "Comparing tube models for predicting the linear rheology of branched polymer melts," J Rheol 54, 223-260 (2010).

[23] Inoue, T., H. Okamoto and K. Osaki, "Birefringence of Amorphous Polymers .1. Dynamic Measurement on Polystyrene," Macromolecules 24, 5670-5675 (1991).

[24] Inoue, T., Y. Mizukami, H. Okamoto, H. Matsui, H. Watanabe, T. Kanaya and K. Osaki, "Dynamic birefringence of vinyl polymers," Macromolecules 29, 6240-6245 (1996).

[25] Inoue, T., H. Matsui and K. Osaki, "Molecular Origin of Viscoelasticity and Chain Orientation of Glassy Polymers," Rheol Acta 36, 239-244 (1997).

[26] Benallal, A., G. Marin, J. P. Montfort and C. Derail, "Linear Viscoelasticity Revisited the Relaxation Function of Monodisperse Polymer Melts," Macromolecules 26, 72297235 (1993).

[27] Leonardi, F., J. C. Majeste, A. Allal and G. Marin, "Rheological models based on the double reptation mixing rule: The effects of a polydisperse environment," J Rheol 44, 675-692 (2000).

[28] Kawasaki, Y., H. Watanabe and T. Uneyama, "A Note for Kohlrausch-Williams-Watts Relaxation Function," Nihon Reoroji Gakk 39, 127-131 (2011). 
[29] Chen, Q., G. J. Tudryn and R. H. Colby, "lonomer Dynamics and the Sticky Rouse Model," J Rheol 57, 1441-1462 (2013).

[30] Chen, Q., S. Liang, H.-S. Shiau and R. H. Colby, "Linear Viscoelastic and Dielectric Properties of Phosphonium Siloxane lonomers," ACS Macro Letters 2, 970-974 (2013).

[31] De Rosa, C., O. R. de Ballesteros, F. Auriemma, R. Di Girolamo, C. Scarica, G. Giusto, S. Esposito, S. Guidotti and I. Camurati, "Polymorphic Behavior and Mechanical Properties of Isotactic 1-Butene-Ethylene Copolymers from Metallocene Catalysts," Macromolecules 47, 4317-4329 (2014).

[32] Fox, T. G., "Influence of diluent and of copolymer composition on the glass temperature of a polymer system," Bull. Am. Phys. Soc. 1, 123-125 (1956).

[33] Di Marzio, E. A., "The glass temperature of polymer blends," Polymer 31, 2294-2298 (1990).

[34] Fetters, L. J., D. J. Lohse, C. A. Garcia-Franco, P. Brant and D. Richter, "Prediction of melt state poly(alpha-olefin) rheological properties: The unsuspected role of the average molecular weight per backbone bond," Macromolecules 35, 10096-10101 (2002).

[35] Kapnistos, M. and D. Vlassopoulos, "Rheological master curves of crystallizing polymer mixtures," Appl Rheol 16, 132-135 (2006).

[36] Liu, C., Z. Zhang, S. Huang and Q. Chen, "Form II to I transformation of polybutene-1 and copolymer of butene-1 and ethylene: A role of amorphous phase," Polymer 149, 146-153 (2018).

[37] Derosa, M. E. and H. H. Winter, "The Effect of Entanglements on the Rheological Behavior of Polybutadiene Critical Gels," Rheol Acta 33, 220-237 (1994).

[38] Larson, R. G., "Combinatorial rheology of branched polymer melts," Macromolecules 34, 4556-4571 (2001).

[39] Park, S. J., S. Shanbhag and R. G. Larson, "A hierarchical algorithm for predicting the linear viscoelastic properties of polymer melts with long-chain branching," Rheol Acta 44, 319-330 (2005).

[40] Milner, S. T. and T. C. B. Mcleish, "Reptation and Contour-Length Fluctuations in Melts of Linear Polymers," Phys. Rev. Lett. 81, 725-728 (1998).

[41] Milner, S. T., T. C. B. McLeish, R. N. Young, A. Hakiki and J. M. Johnson, "Dynamic dilution, constraint-release, and star-linear blends," Macromolecules 31, 9345-9353 (1998).

[42] Milner, S. T. and T. C. B. McLeish, "Parameter-free theory for stress relaxation in star polymer melts," Macromolecules 30, 2159-2166 (1997).

[43] Marrucci, G., "Relaxation by Reptation and Tube Enlargement - a Model for Polydisperse Polymers," J Polym Sci Pol Phys 23, 159-177 (1985).

[44] Ball, R. C. and T. C. B. Mcleish, "Dynamic Dilution and the Viscosity of Star Polymer Melts," Macromolecules 22, 1911-1913 (1989).

[45] Viovy, J. L., M. Rubinstein and R. H. Colby, "Constraint Release in Polymer Melts Tube Reorganization Versus Tube Dilation," Macromolecules 24, 3587-3596 (1991).

[46] Das, C., N. J. Inkson, D. J. Read, M. A. Kelmanson and T. C. B. McLeish, "Computational linear rheology of general branch-on-branch polymers," J Rheol 50, 207-235 (2006).

[47] Park, S. J. and R. G. Larson, "Long-chain dynamics in binary blends of monodisperse linear polymers," J Rheol 50, 21-39 (2006). 
[48] Park, S. J. and R. G. Larson, "Tube dilation and reptation in binary blends of monodisperse linear polymers," Macromolecules 37, 597-604 (2004). 